\title{
Interval-Valued Logic Metric Spaces
}

\author{
Zhao-Rui XUE ${ }^{1, a}$, Min-Xia LUO ${ }^{2, b, *}$ \\ ${ }^{1}$ School of Marxism Studies, China Jiliang University, Hangzhou 310018, PR China \\ ${ }^{2}$ School of Science, China Jiliang University, Hangzhou 310018, PR China \\ akjxzr@163.com, ${ }^{b}$ minxialuo@163.com \\ *Corresponding author: Min-Xia LUO
}

Keywords: Interval-valued fuzzy sets, Distance metric, Logic metric spaces.

\begin{abstract}
A new distance metric between interval-valued fuzzy sets is proposed. And based on this metric, we analysis and compare the structures of four special interval-valued fuzzy metric spaces, which are induced by four well-known residual implication operations. It was proved that the interval-valued fuzzy metric spaces induced by Lucasiewicz implication and Goguen implication are more suitable for interval-valued fuzzy reasoning.
\end{abstract}

\section{Introduction}

Let $M$ be an inference mechanism, and $A, B$ the input and the output of the inference mechanism $M$ respectively. If small disturbance of input $A$ without causing large changes of output $B$ in inference process, then we say that the inference mechanism $M$ has a good robustness. Researchers in different areas have different answers on the concepts of disturbance. Therefore, various related notions such as the largest perturbation, the average perturbation, $\delta$-equalities, $\delta$-sensitivity, largest $\delta$-sensitivity, average $\delta$-sensitivity and so on were proposed. The largest perturbation and the average perturbation of fuzzy sets were proposed and the perturbation of several fuzzy reasoning systems were discussed in [16]. Cai [1] discussed the robustness of fuzzy inference using $\delta$-equalities of connectives and fuzzy implication operators. Literature [7,9] proposed concepts of $\delta$-sensitivity, largest $\delta$-sensitivity and average $\delta$-sensitivity of fuzzy connectives, and discussed the robustness of fuzzy reasoning. By comparing and analyzing these concepts, we notice that these concepts are based on different distance metrics. It is well known that fuzzy connectives determine the internal structure of a fuzzy logic system. However, the construction of distance metrics does not involve fuzzy connectives in previous works. Taking this into account, Dai et al. [3] and Jin etal. [4] proposed the notion of logic similarity degree between fuzzy sets based on fuzzy connectives, and discuss robustness of fuzzy reasoning. Wang et al. [15] proposed a new distance metric based on residuated implication and conjunction connective, and discussed the robustness of full implication triple I inference method. Then Duan [5] studied the structures of four specific logic metric.

However, there are some limitations when we deal with imprecise information using fuzzy sets. Therefore, interval-valued fuzzy set was introduced by Zadeh [17], which can not only effectively reduce the loss of fuzzy information but also reflect the vagueness and uncertainty in information processing. And then many researchers have studied this topic and extended approximate inference to the case of interval-valued fuzzy sets. Li et al. [8] extended CRI method to the case of interval-valued fuzzy set and discussed the robustness of interval-valued CRI method. Luo et al. [11, 12] extended triple I method and reverse triple I method to the case of interval-valued fuzzy sets and studied their robustness respectively. These researches of robustness of interval-valued fuzzy reasoning methods are based on moore metric.

However, it is easy to lose information when we use moore metric to study interval-valued fuzzy inference. For example, suppose $S I(X)$ is the class of all interval-valued fuzzy subsets of non-empty sets $X$. Let $X=\left\{x_{1}, x_{2}, \ldots, x_{10}\right\}$, for $A, B$ and $C \in S I(X), A\left(x_{i}\right)=[1,1](1 \leq i \leq 10)$, $B\left(x_{i}\right)=[1,1](1 \leq i \leq 9), B\left(x_{10}\right)=[1,1]$ and $C\left(x_{i}\right)=[0,0](1 \leq i \leq 10)$. If we use the moore metric, 
then we obtain $\left.\quad d(A, B)=\max \operatorname{nax}_{i}\left\{\left|A_{l}\left(x_{i}\right)-B_{l}\left(x_{i}\right)\right|,\left|A_{r}\left(x_{i}\right)-B_{r}\left(x_{i}\right)\right|\right\}\right\}=1 \quad$ and $\left.d(A, C)=\max \max _{i}\left\{\left|A_{l}\left(x_{i}\right)-C_{l}\left(x_{i}\right)\right|,\left|A_{r}\left(x_{i}\right)-C_{r}\left(x_{i}\right)\right|\right\}\right\}=1$. Obviously, this result is not reasonable. And because the behavior of a fuzzy logic system is mainly determined by its fuzzy connectives and fuzzy implication operators, based on this point, we propose a new distance metric of interval-valued fuzzy sets based on left-continuous t-norm and its residual implication. We analyze and compare the structures of four specific interval-valued fuzzy metric spaces.

The remaining part is organized as the follows. Section 2 review some concepts needed for the paper. In Section 3, a new distance between interval-valued fuzzy sets based on left-continuous t-norm and its resituated implication is proposed, and four interval-valued fuzzy metric spaces $(S I(X), d)$ are given. In Section 4, four interval-valued fuzzy metric spaces are study. The final Section includes our conclusions.

\section{Preliminaries}

In this section, we review some concepts that will be required for our following work. Let $S E\{[x, y] \mid x \leq y, x, y \in[0,1]\}$. An ordering on $S I$ as $[a, b] \leq[c, d]$ if $a \leq c$ and $b \leq d$ is called component-wise order or Kulisch-Miranker order [2]. It is easy to verify that the ordering just defined is a partially ordering on $S I$. Furthermore, take $[a, b] \wedge[c, d]=[a, b] \operatorname{iff}[a, b] \leq[c, d]$ and $[a, b] \vee[c, d]=[c, d]$ iff $[a, b] \leq[c, d]$. In this paper, let $X=\left\{x_{1}, x_{2}, \ldots, x_{10}\right\}$ be non-empty sets, $S I(X)$ denote interval-valued fuzzy subsets of non-empty sets $X$, for $1 \leq i \leq n, A\left(x_{i}\right) \in S I(X)$ ( $A\left(x_{i}\right)$ denoted by $\left.\left[A_{l}\left(x_{i}\right), A_{r}\left(x_{i}\right)\right]\right) . A^{c}$ is complement of interval-valued $A$, where $A^{c}=\left[1-A_{r}, 1-A_{l}\right]$.

Definition 2.1 ([6]) A function $T:[0,1]^{2} \rightarrow[0,1]$ is called a triangular norm (t-norm) if it commutative, associative, non-decreasing in each argument and $T(x, 1)=x$ for all $a \in[0,1]$.

Definition 2.2 ([13]) Let $L$ be a bounded lattice. $T$ is a t-norm on $L$ If there exists another operator $R_{T}: L^{2} \rightarrow L$ such that $T(a, b) \leq c$ if and only if $a \leq R_{T}(b, c)$; for all $a, b, c \in L$; then $R_{T}$ is called the residuum of $T,\left(T, R_{T}\right)$ is called a residuated pair on $L$.

Example 2.1 ([14]) Defined on the unit interval [0,1] that:

(1) Minimum t-norm and its residuum, Gödel implication:

$$
T_{G}(a, b)=a \wedge b, \quad R_{G}(a, b)=\left\{\begin{array}{l}
1, i f a \leq b, \\
b, \text { if } a>b .
\end{array}\right.
$$

(2) Nilpotent minimum t-norm and its residuum, $R_{0}$ implication:

$$
T_{0}(a, b)=\left\{\begin{array}{l}
a \wedge b, \text { if } a+b>1, \\
0, \text { otherwise. }
\end{array} \quad R_{0}(a, b)=\left\{\begin{array}{l}
1, \text { if } a \leq b, \\
(1-a) \vee b, \text { otherwise } .
\end{array}\right.\right.
$$

(3) Product t-norm and its residuum, Goguen implication:

$$
T_{G_{0}}(a, b)=a b, \quad R_{G_{0}}(a, b)=\left\{\begin{array}{l}
1, \text { if } a \leq b, \\
\frac{b}{a}, \text { if } a>b .
\end{array}\right.
$$

(4) Lucasiewicz t-norm and its residuum, Lucasiewicz implication:

$$
T_{L}(a, b)=0 \vee(a+b-1), \quad R_{L}(a, b)=1 \wedge(1-a+b) .
$$


Definition 2.3 ([10]) A real function $d: F(X) \times F(X) \rightarrow[0,1]$ is called a distance, if $d$ satisfied the following properties:

$(D 1): d(A, B)=d(B, A)$ for all $A, B \in F(X) ;(D 2): d\left(A, A^{c}\right)=1 \Leftrightarrow A \in P(X)$;

(D3): $\quad d(A, B)=0$ if and only if $A=B$, for all $A, B \in F(X)$;

(D4): for all $A, B, C \in F(X)$, if $A \leq B \leq C$, then $d(A, C) \geq d(A, B)$ and $d(A, C) \geq d(B, C)$. Where $F(X)$ is fuzzy subsets of non-empty set $X$ and $P(X)$ stands for the sets of all crisp sets in $X$.

\section{A New Distance between Interval-Valued Fuzzy Sets}

Definition 3.1 Let $X=\left\{x_{1}, x_{2}, \ldots, x_{n}\right\}, A, B \in S I(X)$. Supspose $T$ is a left-continuous t-norm and $R_{T}$ is the corresponding residuated implication. Let $d(A, B)=1-\frac{1}{n} \sum_{i=1}^{n} T\left(\rho\left(A\left(x_{i}\right), B\left(x_{i}\right)\right), \rho\left(B\left(x_{i}\right), A\left(x_{i}\right)\right)\right)$, where $\rho\left(A\left(x_{i}\right), B\left(x_{i}\right)\right)=\min \left\{R_{T}\left(A_{l}\left(x_{i}\right), B_{l}\left(x_{i}\right)\right), R_{T}\left(A_{r}\left(x_{i}\right), B_{r}\left(x_{i}\right)\right)\right\}$. Then $d$ is called a distance metric on $\operatorname{SI}(X)$ and $(S I(X), d)$ is called a logic metric space.

Theorem $3.1 d$ defined by Definition 3.1 is a distance metric on $\operatorname{SI}(X)$.

Proof:(D1):Due to $T\left(\rho\left(A\left(x_{i}\right), B\left(x_{i}\right)\right), \rho\left(B\left(x_{i}\right), A\left(x_{i}\right)\right)\right)=T\left(\rho\left(B\left(x_{i}\right), A\left(x_{i}\right)\right), \rho\left(A\left(x_{i}\right), B\left(x_{i}\right)\right)\right)$

Then $d(A, B)=d(B, A)$.

(D2): Suppose for $1 \leq i \leq n, A\left(x_{i}\right)=[0,0]$ and $A^{c}\left(x_{i}\right)=[1,1]$, then $\rho\left(A\left(x_{i}\right), A^{c}\left(x_{i}\right)\right)=1$. And $\rho\left(A^{c}\left(x_{i}\right), A\left(x_{i}\right)\right)=0$. Thus we have $d\left(A, A^{c}\right)=1-\frac{1}{n} \sum_{i=1}^{n} T[1,0]=1$. Similarly, we can prove that if $A\left(x_{i}\right)=[1,1](1 \leq i \leq n)$, then $d\left(A, A^{c}\right)=1$. If $d\left(A, A^{c}\right)=1$, then by Definition 3.1, we can obtain $\frac{1}{n} \sum_{i=1}^{n} T\left(\rho\left(A\left(x_{i}\right), A^{c}\left(x_{i}\right)\right), \rho\left(A^{c}\left(x_{i}\right), A\left(x_{i}\right)\right)\right)=0$,

i.e. $\quad T\left(\rho\left(A\left(x_{i}\right), A^{c}\left(x_{i}\right)\right), \rho\left(A^{c}\left(x_{i}\right), A\left(x_{i}\right)\right)\right)=0 \quad$, then $\quad \rho\left(A\left(x_{i}\right), A^{c}\left(x_{i}\right)\right)=0 \quad$ or $\rho\left(A^{c}\left(x_{i}\right), A\left(x_{i}\right)\right)=0$. If $\rho\left(A\left(x_{i}\right), A^{c}\left(x_{i}\right)\right)=0$, then $A_{l}\left(x_{i}\right)=1$ and $A_{l}^{c}\left(x_{i}\right)=0$, i.e., $A_{l}\left(x_{i}\right)=[1,1]$. Similarly, if $\rho\left(A^{c}\left(x_{i}\right), A\left(x_{i}\right)\right)=0$, then $A\left(x_{i}\right)=[0,0]$. Therefore, if $d\left(A, A^{c}\right)=1$ then $A \in P(X)$.

(D4): If $A \leq B \leq C$, then $A_{l}\left(x_{i}\right) \leq B_{l}\left(x_{i}\right) \leq C_{l}\left(x_{i}\right)$ and $A_{r}\left(x_{i}\right) \leq B_{r}\left(x_{i}\right) \leq C_{r}\left(x_{i}\right)$, i.e., $\rho\left(B\left(x_{i}\right), A\left(x_{i}\right)\right) \leq \rho\left(C\left(x_{i}\right), A\left(x_{i}\right)\right) \quad$ and $\quad \rho\left(C\left(x_{i}\right), A\left(x_{i}\right)\right) \leq \rho\left(C\left(x_{i}\right), B\left(x_{i}\right)\right) \quad$. Thus, $d(A, C) \geq d(A, B)$ and $d(A, C) \geq d(B, C)$. Therefore, $d$ is a distance on $\operatorname{SI}(X)$.

Proposition 3.1 (1) If $R$ is Gödel implication and $T$ is corresponding t-norm, then,

$$
d_{G}(A, B)=1-\frac{1}{n} \sum_{i=1}^{n}\left\{\rho_{G}\left(A\left(x_{i}\right), B\left(x_{i}\right)\right) \wedge \rho_{G}\left(B\left(x_{i}\right), A\left(x_{i}\right)\right)\right\}
$$

(2) If $R$ is $R_{0}$ implication and $T$ is corresponding t-norm, then,

$$
\left.d_{0}(A, B)=1-\frac{1}{n} \sum_{i=1}^{n} T_{0}\left(\rho_{0}\left(A\left(x_{i}\right), B\left(x_{i}\right)\right)+\rho_{0}\left(B\left(x_{i}\right), A\left(x_{i}\right)\right)-1\right) \vee 0\right\}
$$

(3) If $R$ is Goguen implication and $T$ is corresponding t-norm, then 


$$
d_{G_{0}}(A, B)==1-\frac{1}{n} \sum_{i=1}^{n}\left\{\left(\frac{B_{l}\left(x_{i}\right)}{A_{l}\left(x_{i}\right)} \wedge \frac{B_{r}\left(x_{i}\right)}{A_{r}\left(x_{i}\right)} \wedge 1\right) \cdot\left(\frac{A_{l}\left(x_{i}\right)}{A_{l}\left(x_{i}\right)} \wedge \frac{A_{r}\left(x_{i}\right)}{B_{r}\left(x_{i}\right)} \wedge 1\right)\right\} .
$$

(4) If $R$ is Lucasiewicz implication and $T$ is corresponding t-norm, then,

$$
d_{L}(A, B)=1-\frac{1}{n} \sum_{i=1}^{n}\left\{\left(\rho_{L}\left(A\left(x_{i}\right), B\left(x_{i}\right)\right)+\rho_{L}\left(B\left(x_{i}\right), A\left(x_{i}\right)\right)-1\right) \vee 0\right\} .
$$

By above four interval-valued distance metrics, we can construct four interval-valued fuzzy metric spaces $\left(S I(X), d_{G}\right),\left(S I(X), d_{0}\right),\left(S I(X), d_{G_{0}}\right)$ and $\left(S I(X), d_{L}\right)$, respectively.

\section{Analysis of Four Interval-Valued Fuzzy Metric Spaces Structures}

In a metric space $(Y, d)$, let $y \in Y$. For arbitrary $0<\varepsilon<1$, if we could always find a another point $y^{\prime} \in Y \operatorname{such}$ that $d\left(y, y^{\prime}\right)<\varepsilon$, then we say that $y$ is a condensation point of $Y$. Otherwise, if exists $\delta>0$ such that for every $y^{\prime} \in Y, d\left(y, y^{\prime}\right) \geq \delta$, then we say that $y$ is an isolated point. In this section, we analyze condensation points and isolated points in these four metric spaces $\left(S I(X), d_{G}\right),\left(S I(X), d_{0}\right),\left(S I(X), d_{G_{0}}\right)$ and $\left(S I(X), d_{L}\right)$ respectively. Let $X=\left\{x_{1}, x_{2}, \ldots, x_{n}\right\}$, $A \in \operatorname{SI}(X)$, if there exists $x_{k} \in X$, such that $A\left(x_{k}\right)=[1,1]$, then $A$ is a normal interval-valued fuzzy set.

Theorem 4.1 Let $X=\left\{x_{1}, x_{2}, \ldots, x_{n}\right\}$ and $A \in S I(X)$. Then $A$ is condensation point of $\left(S I(X), d_{G}\right)$ if and only if $A$ is normal interval-valued fuzzy set on $X$.

Proof : Let $A$ be normal interval-valued fuzzy set, then there exists $x_{k} \in X$ such that $A\left(x_{i}\right)=[1,1]$. For all $\varepsilon \in(0,1)$, take $B\left(x_{k}\right)=[1-\varepsilon, 1-\varepsilon], B\left(x_{j}\right)=A\left(x_{j}\right)(j \neq k)$. It is obvious that $B \neq A$ and $d_{G}(A, B)=1-\frac{1}{n} \sum_{i=1}^{n} T\left(\rho\left(A\left(x_{i}\right), B\left(x_{i}\right)\right), \rho\left(B\left(x_{i}\right), A\left(x_{i}\right)\right)\right)$

$=1-\frac{1}{n} \sum_{i=1}^{n} T\left(\min \left\{R_{G}\left(A_{l}\left(x_{i}\right), B_{l}\left(x_{i}\right)\right), R_{G}\left(A_{r}\left(x_{i}\right), B_{r}\left(x_{i}\right)\right)\right\}, \min \left\{R_{G}\left(B_{l}\left(x_{i}\right), A_{l}\left(x_{i}\right)\right), R_{G}\left(B_{r}\left(x_{i}\right), A_{r}\left(x_{i}\right)\right)\right\}\right)$ $=1-\frac{1}{n}((n-1)+(1-\varepsilon))=\frac{\varepsilon}{n}<\varepsilon$. Therefore, there always exists an interval-valued fuzzy set $B$ which is different from $A$ in the arbitrary neighborhood of $A$, i.e. $d(A, B)<\varepsilon$. Thus, $A$ is condensation point in the metric space $\left(S I(X), d_{G}\right)$.

If $A$ is not normal, then $A\left(x_{i}\right) \neq[1,1]$ for all $x_{i} \in X$. Take arbitrary $B \in \operatorname{SI}(X)$ such that $B \neq A$, then there exists $x_{k} \in X$ such that $B\left(x_{k}\right) \neq A\left(x_{k}\right)$ and $A_{l}\left(x_{k}\right)<B_{l}\left(x_{k}\right)$. We can easily prove that $T\left(\rho\left(A\left(x_{k}\right), B\left(x_{k}\right)\right), \rho\left(B\left(x_{k}\right), A\left(x_{k}\right)\right)\right) \leq A_{l}\left(x_{k}\right)$. Moreover, when $A\left(x_{i}\right)=B\left(x_{i}\right)(i \neq k)$, then $T\left(\rho\left(A\left(x_{i}\right), B\left(x_{i}\right)\right), \rho\left(B\left(x_{i}\right), A\left(x_{i}\right)\right)\right)=1$. Thus, suppose there only exists $x_{k} \in X$ such that $B\left(x_{k}\right) \neq A\left(x_{k}\right)=\left[a_{1}, a_{2}\right]\left(a_{1} \neq 1\right)$, Then $d_{G}(A, B)=1-\frac{1}{n} \sum_{i=1}^{n} T\left(\rho\left(A\left(x_{i}\right), B\left(x_{i}\right)\right), \rho\left(B\left(x_{i}\right), A\left(x_{i}\right)\right)\right)$ $\geq 1-\frac{1}{n}\left((n-1)+a_{1}\right)=\frac{1}{n}\left(1-a_{1}\right)=\delta>0$. As a result, there exists $\delta>0$, such that there is no interval-valued fuzzy set different from $A$ in the $\delta$ neighborhood of $A$, i.e. $A$ is an isolated point of $\left(S I(X), d_{G}\right)$. 
Theorem 4.2 Let $X=\left\{x_{1}, x_{2}, \ldots, x_{n}\right\}$ and $A \in S I(X)$. Then $A$ is condensation point of $\left(\operatorname{SI}(X), d_{0}\right)$ if and only if $A$ or $A^{c}$ is normal interval-valued fuzzy set on $X$.

Proof: Assume that $A$ and $A^{c}$ are both not normal interval-valued fuzzy set. Then for all $x_{i} \in X$, $A\left(x_{i}\right) \neq[1,1]$ and $A\left(x_{i}\right) \neq[0,0]$. Take arbitrary $B \in S I(X)$ such that $B \neq A$, then there exists $x_{k} \in X$, such that $B\left(x_{k}\right) \neq A\left(x_{k}\right)=\left[a_{1}, a_{2}\right]\left(\left[a_{1}, a_{2}\right] \neq[1,1]\right.$ and $\left.\left[a_{1}, a_{2}\right] \neq[0,0]\right)$. We can easily prove that $T\left(\rho\left(A\left(x_{k}\right), B\left(x_{k}\right)\right), \rho\left(B\left(x_{k}\right), A\left(x_{k}\right)\right)\right) \leq a_{1} \vee a_{1}^{c}$. Furthermore, when $B\left(x_{i}\right)=A\left(x_{i}\right)(i \neq k)$, then $T\left(\rho\left(A\left(x_{i}\right), B\left(x_{i}\right)\right), \rho\left(B\left(x_{i}\right), A\left(x_{i}\right)\right)\right)=1$. Thus, suppose there only exists $x_{k} \in X$ such that $B\left(x_{k}\right) \neq A\left(x_{k}\right)=\left[a_{1}, a_{2}\right]\left(\left[a_{1}, a_{2}\right] \neq[1,1]\right.$ and $\left.\left[a_{1}, a_{2}\right] \neq[0,0]\right)$, hence

$$
d_{0}(A, B)=1-\frac{1}{n} \sum_{i=1}^{n} T\left(\rho\left(A\left(x_{i}\right), B\left(x_{i}\right)\right), \rho\left(B\left(x_{i}\right), A\left(x_{i}\right)\right)\right) \geq 1-\frac{1}{n}\left((n-1)+a_{1} \vee\left(1-a_{1}\right)\right) \geq \delta>0 \quad \text {.As } \quad \text { a }
$$

result, there is no interval-valued fuzzy set different from $A$ in the $\delta>0$ neighborhood of $A$. Hence, $A$ is isolated point of $\left(\operatorname{SI}(X), d_{0}\right)$.

Secondly, if $A$ is normal, then $A$ is a condensation point of $\left(\operatorname{SI}(X), d_{0}\right)$. The proof is similar to that of Theorem 4.1. If $A^{c}$ is normal, then there exists $x_{k}$ such that $A\left(x_{k}\right)=[0,0]$. For all $\varepsilon \in(0,1)$, take $B \in S I(X)$ such that $B\left(x_{k}\right)=[\varepsilon, \varepsilon], B\left(x_{j}\right)=A\left(x_{j}\right)(j \neq k)$.

Then $T\left(\rho\left(A\left(x_{k}\right), B\left(x_{k}\right)\right), \rho\left(B\left(x_{k}\right), A\left(x_{k}\right)\right)\right)=1-\varepsilon, T\left(\rho\left(A\left(x_{j}\right), B\left(x_{j}\right)\right), \rho\left(B\left(x_{j}\right), A\left(x_{j}\right)\right)\right)=1$.

Thus $d_{0}(A, B)=1-\frac{1}{n} \sum_{i=1}^{n} T\left(\rho\left(A\left(x_{i}\right), B\left(x_{i}\right)\right), \rho\left(B\left(x_{i}\right), A\left(x_{i}\right)\right)\right)=1-\frac{1}{n}((n-1)+1-\varepsilon)=\frac{\varepsilon}{n}<\varepsilon$.

Therefore, $A$ is condensation point of $\left(S I(X), d_{0}\right)$.

Theorem 4.3 Let $X=\left\{x_{1}, x_{2}, \ldots, x_{n}\right\}$ and $A \in S I(X) . A\left(x_{i}\right)=[0,0](1 \leq i \leq n)$ is the only isolated point of $\left(S I(X), d_{G_{0}}\right)$.

Proof: Let $A\left(x_{i}\right)=[0,0], i=1, \ldots, n$. Then for all $B \in S I(X)$ satisfying $B \neq A$, for all $x_{i} \in X$, $B\left(x_{i}\right) \neq[0,0]$ and $A\left(x_{i}\right)=[0,0]$, then we have $T\left(\rho\left(A\left(x_{i}\right), B\left(x_{i}\right)\right), \rho\left(B\left(x_{i}\right), A\left(x_{i}\right)\right)\right)=0$. Hence, $d_{G_{0}}(A, B)=1-\frac{1}{n} \sum_{i=1}^{n} T\left(\rho\left(A\left(x_{i}\right), B\left(x_{i}\right)\right), \rho\left(B\left(x_{i}\right), A\left(x_{i}\right)\right)\right)=1>\varepsilon$. Therefore, $A$ is isolated point of $\left(S I(X), d_{G_{0}}\right)$. If for all $x_{i} \in X, A_{l}\left(x_{i}\right) \neq 0$, then $A_{r}\left(x_{i}\right) \neq 0$, then exists $x_{k} \in X$, such that $A\left(x_{k}\right)=\left[c_{1}, c_{2}\right]\left(c_{1} \neq 0\right)$. Given $\varepsilon>0$ and given $m_{1}>\frac{1}{\varepsilon}, m_{2}>\frac{1}{\varepsilon}$ and $m_{1}<m_{2}$, let $b_{1}=\left(1-\frac{1}{m_{1}}\right) c_{1}$ and $b_{2}=\left(1-\frac{1}{m_{2}}\right) c_{2}$. Let $B \in S I(X)$, such that $B\left(x_{k}\right)=\left[b_{1}, b_{2}\right], B\left(x_{j}\right)=A\left(x_{j}\right)(j \neq k)$, then we have $T\left(\rho\left(A\left(x_{k}\right), B\left(x_{k}\right)\right), \rho\left(B\left(x_{k}\right), A\left(x_{k}\right)\right)\right)=\left(1-\frac{1}{m_{1}}\right) \wedge\left(1-\frac{1}{m_{2}}\right) \quad$ and $T\left(\rho\left(A\left(x_{j}\right), B\left(x_{j}\right)\right), \rho\left(B\left(x_{j}\right), A\left(x_{j}\right)\right)\right)=1$. Thus $d_{G_{0}}(A, B)=1-\frac{1}{n} \sum_{i=1}^{n} T\left(\rho\left(A\left(x_{i}\right), B\left(x_{i}\right)\right), \quad \rho\left(B\left(x_{i}\right), A\left(x_{i}\right)\right)\right)=1-\frac{1}{n}\left((n-1)+\left(1-\frac{1}{m_{1}}\right) \wedge\left(1-\frac{1}{m_{2}}\right)\right)$ $=\frac{1}{n}\left\{\left(\frac{1}{n}-\frac{1}{n m_{1}}\right) \wedge\left(\frac{1}{n}-\frac{1}{n m_{2}}\right)\right\}=\max \left\{\frac{1}{n m_{1}}, \frac{1}{n m_{2}}\right\} \leq \frac{\varepsilon}{n} \leq \varepsilon$. Hence, $A$ is condensation point of $\left(\operatorname{SI}(X), d_{G_{0}}\right)$.

Theorem 4.4 Let $X=\left\{x_{1}, x_{2}, \ldots, x_{n}\right\}$. Then there is no isolated point in the logic metric space $\left(S I(X), d_{L}\right)$. 
Proof : $\forall A(x) \in S I(X), d_{L}(A, B)=1-\frac{1}{n} \sum_{i=1}^{n} T\left(\rho\left(A\left(x_{i}\right), B\left(x_{i}\right)\right), \rho\left(B\left(x_{i}\right), A\left(x_{i}\right)\right)\right)$ $=\frac{1}{n} \sum_{i=1}^{n}\left\{\left(A_{l}\left(x_{i}\right)-B_{l}\left(x_{i}\right)\right) \vee\left(A_{r}\left(x_{i}\right)-B_{r}\left(x_{i}\right)\right)\right\}+\frac{1}{n} \sum_{i=1}^{n}\left\{\left(B_{l}\left(x_{i}\right)-A_{l}\left(x_{i}\right)\right) \vee\left(B_{r}\left(x_{i}\right)-A_{r}\left(x_{i}\right)\right)\right\}$. For all $\varepsilon>0$, we can find $B \in S I(X)$ such that $\left|A_{l}\left(x_{i}\right)-B_{l}\left(x_{i}\right)\right|<\frac{\varepsilon}{2}$ and $\left|A_{r}\left(x_{i}\right)-B_{r}\left(x_{i}\right)\right|<\frac{\varepsilon}{2}(i=1, \ldots, n)$. Then, $d_{L}(A, B)<\varepsilon$ i.e., $A$ is condensation point of $\left(\operatorname{SI}(X), d_{L}\right)$.

\section{Conclusions}

In this paper, a new distance metric between interval-valued fuzzy sets is proposed, which is induced by left-continuous t-norm and corresponding residuated implication. By comparing and analyzing the structures of four special interval-valued fuzzy metric spaces respectively, we proved that there is no isolated point in $\left(\operatorname{SI}(X), d_{L}\right)$, and there is only one isolated point in $\left(\operatorname{SI}(X), d_{G_{0}}\right)$. However, there are too many isolated points in $\left(S I(X), d_{G}\right)$ and $\left(S I(X), d_{0}\right)$. In order to better study interval-valued fuzzy reasoning, we don't expect there are too many isolated points in interval-valued fuzzy metric spaces. Therefore, interval-valued fuzzy metric spaces $\left(S I(X), d_{L}\right)$ and $\left(S I(X), d_{G_{0}}\right)$ are more suitable for interval-valued fuzzy reasoning.

\section{Acknowledgement}

This research was supported by the National Natural Science Foundation of China (No.61773019).

\section{References}

[1] K.Y. Cai, Robustness of fuzzy reasoning and $\delta$-equalities of fuzzy sets, IEEE Trans Fuzzy Syst, 9(2001) 738-750.

[2] B.A. Davey, H.A. Priestley, Introduction to Lattices and Order, Cambridge University Press, Cambridge, 1990.

[3] S.S. Dai, D.W. Pei, D.H. Guo, Robustness analysis of full implication inference method, International Journal of Approximate Reasoning, 54 (2013) 653-666.

[4] J.H. Jin, Y.M. Li, C.Q. Li, Robustness of fuzzy reasoning via logically equivalence measure, Information Sciences. 177(2007), 5103-5117.

[5] J.Y. Duan, Y.M. Li, Robustness analysis of logic metrics on $F(X)$, International Journal of Approximate Reasoning, 61(2015) 33-42.

[6] E. Klement, R. Mesiar, E. Pap, Triangular Norms, Kluwer Academic Publishers, Dordrecht, 2000.

[7] Y.M. Li, Analysis of Fuzzy System, Science Press, Beijing, 2005.

[8] D.Ch. Li, Y.M.Li, Y.J.Xie. Robustness of interval-valued fuzzy inference, Information Sciences, $181(2011)$ 4754-4764.

[9] Y.M. Li, D.Ch. Li, J.J. W, et.al, An approach of measure the robustness of fuzzy reasoning, Int J Intell Syst, 20(2005) 393-413.

[10] X.C. Liu, Entropy, distance measure and similarity measure of fuzzy sets and their relations, Fuzzy Sets and Systems, 52(1992), pp. 305-318.

[11] M.X. Luo, K. Zhang, Robustness of full implication argorithms based on interval-valued fuzzy 
inference, International Journal of Approximate Reasoning, 62(2015) 61-72.

[12] M.X. Luo, X.L. Zhou, Robustness of reverse triple I algorithms based on interval-valued fuzzy inference, International Journal of Approximate Reasoning, 66(2015) 16-26.

[13] G.J. Wang, Non-classical Mathematical Logic and Approximate Reasoning. Science Press, Beijing, 2000(in Chinese).

[14] G.J. Wang, H.J. Zhou, Introduction to Mathematical Logic and Resolution Principle. Science Press/U.K. Alpha Science International Limited, Beijing/Oxford, 2009.

[15] G.J. Wang, J.Y. Duan, On robustness of the full implication triple I inference method with respect to finer measurements. International Journal of Approximate Reasoning, 55(2014) 787-796.

[16] M.S. Ying, Perturbation of fuzzy reasoning, IEEE Trans Fuzzy Syst, 7(1999) 625-629.

[17] L.A. Zadeh, Theory of approximate reasoning, in: J.E. Hayes, D.Michie, L.I. Mikulich(Eds.), Machine Intelligence, Ellis Horwood, Chichester, 1970, pp. 149-194. 